\title{
Directed Graphs of Entanglement Two
}

\author{
Erich Grädel, Łukasz Kaiser, and Roman Rabinovich \\ Mathematische Grundlagen der Informatik, RWTH Aachen University \\ \{graedel, kaiser, rabinovich\}@logic.rwth-aachen. de
}

\begin{abstract}
Entanglement is a complexity measure for directed graphs that was used to show that the variable hierarchy of the propositional modal $\mu$-calculus is strict. While graphs of entanglement zero and one are indeed very simple, some graphs of entanglement two already contain interesting nesting of cycles. This motivates our study of the class of graphs of entanglement two, as these are both simple in a sense and already complex enough for modelling certain structured systems.

Undirected graphs of entanglement two were already studied by Belkhir and Santocanale and a structural decomposition for such graphs was given. We study the general case of directed graphs of entanglement two and prove that they can be decomposed as well, in a way similar to the known decompositions for tree-width, DAG-width and Kelly-width. Moreover, we show that all graphs of entanglement two have Kelly-width at most three. It is known that such graphs also have DAG-width at most three [2]. Since there exist both graphs with DAG-width three and graphs with Kelly-width three, but with arbitrary high entanglement, this confirms that graphs of entanglement two are a very basic class of graphs with cycles intertwined in an interesting way.
\end{abstract}

\section{Introduction}

In recent years, several parameters have been proposed to measure the structural complexity of directed graphs in a similar way as tree-width measures the complexity of undirected ones. While tree-width indicates how closely a graph resembles a tree, the intuition behind complexity measures for directed graphs is that acyclic graphs are simple, and that we can measure the complexity of a graph by the extent to which its cycles are intertwined, or entangled. It has turned out that there are several different ways to make this intuition precise, and several methods to obtain such complexity measures. The two main methodologies are appropriate decompositions of the graph, similar to tree decompositions, and graph searching games, also called robber-and-cops games, where a number of cops try to catch a fugitive on the graph. The movements of the fugitive are restricted by the edges of the graph, and the number of cops that are necessary to catch the fugitive determine the complexity of the graph.

DAG-width, introduced in $[2,11]$ is defined by DAG-decompositions. A DAGdecomposition of width $k$ of a graph $\mathcal{G}$ is given by a directed acyclic graph $(\mathrm{DAG}) \mathcal{D}$ and a map that associates with every node of the DAG a set of 
at most $k$ vertices of $\mathcal{G}$, covering the entire graph $\mathcal{G}$ in such a way that for every $d \in \mathcal{D}$, the edges of $\mathcal{G}$ leaving a node strictly below $d$ are guarded by vertices in $d$. DAG-width can also be characterised by a variant of a graph searching game (the directed cops and visible robber game), but with the somewhat unsatisfactory restriction that the cops are only allowed to use robber-monotone strategies, i.e. a move of the cops must never enlarge the portion of the graph in which the robber can move. It has recently been proved [10] that this restriction is necessary. Indeed, there exist families of graphs on which the difference between the DAG-width and the number of cops that can catch the robber with a non-monotone strategy is unbounded.

Kelly-width, see [8], is a similar measure that can either be defined by a somewhat refined notion of decomposition, called Kelly-decompositions, or by a graph searching game in which the robber is invisible for the cops, and inert, i.e. he can move only when a cop is about to land on his current position. Again, the correspondence between decompositions and games only holds with the restriction to monotone strategies [10].

Entanglement, introduced in [3], has been motivated by applications concerning the modal $\mu$-calculus and parity games. It is defined by the entanglement game, in which the movements of both cops and robber are more restricted than in other graph searching games. In each move the cops either stay where they are or place one of them on the current position of the robber. The robber then moves, along an edge to a new vertex that must not be occupied by a cop. If no such vertex exists, the robber is captured. Here, strategies need not be monotone.

Entanglement is in a sense more delicate than tree-width, DAG-width, or Kelly-width [8]. There exist graphs with tree-width two and arbitrary large entanglement, as well as graphs with DAG-width two and unbounded entanglement. There exist a number of other measures for directed graphs, including directed tree-width [9], pathwidth, cycle rank [6], D-width [13]. For surveys over different measures, we refer to $[7,12]$.

The strengths of the entanglement measure are the close connection with modal logics and bisimulation invariant properties, and the natural game-theoretic characterisation. Entanglement has been a crucial ingredient in the proof that the variable hierarchy of the modal $\mu$-calculus is strict [4]. Further, it has been proved that parity games can be efficiently solved on game graphs with bounded entanglement ${ }^{1}$. The entanglement does not increase when we take bisimulation quotients, and as a consequence of this observation it has been proved that winning regions of parity games are definable in least fixed point logic on graphs of bounded entanglement [5].

The main weakness of the entanglement measure (at the current state of the art) is that it does not come with a natural notion of decomposition, such as the ones for tree-width, DAG-width, or Kelly-width. Decompositions are crucial for algorithmic applications, since they allow to break the structure into

\footnotetext{
${ }^{1}$ An analogous result also holds for bounded DAG-width and bounded Kelly-width.
} 
smaller parts and process these in a systematic way. A structural characterisation of entanglement has been given in [4] in terms of the minimal feedback of the finite unravellings of the graph to a finite tree with back-edges. However, while this produces a game-free definition of entanglement, it does not give a decomposition.

In this paper we study graphs of entanglement two. While graphs of entanglement zero and one are indeed very simple, graphs of entanglement two may already have an arbitrary nesting of cycles, and they are rich enough to model interesting classes of structured systems. We provide structural characterisations of this class, and find appropriate decompositions, similar to the ones for tree-width, DAG-width, and Kelly-width. Moreover, we show that all graphs of entanglement two have Kelly-width at most three. It is known that such graphs also have DAG-width at most three [2]. Since there exist both graphs with DAG-width three and graphs with Kelly-width three, but with arbitrary high entanglement, this confirms that graphs of entanglement two are a very basic class of graphs with cycles intertwined in an interesting way.

\section{Entanglement}

In this paper, a graph is always meant to be finite and directed. To deal with undirected graphs, we view undirected edges $\{u, v\}$ as pairs $(u, v)$ and $(v, u)$ of directed edges, so undirected graphs are directed graphs with a symmetric edge relation.

For a graph $\mathcal{G}=(V, E)$ and $V^{\prime} \subseteq V$, we write $\mathcal{G}\left[V^{\prime}\right]$ to denote the subgraph of $\mathcal{G}$ induced by the vertex set $V^{\prime}$. For a vertex $a \in V$, we write $\mathcal{G} \backslash a$ for $\mathcal{G}[V \backslash\{a\}]$. Further, let $v E:=\{w \in V:(v, w) \in E\}$.

Entanglement is defined by way of the entanglement game $\mathrm{EG}_{k}(\mathcal{G})$, played by a robber against $k$ cops on a directed graph $\mathcal{G}$. Initially, all cops are outside the graph and the robber selects an arbitrary starting vertex $v_{0}$ of $\mathcal{G}$. The players move in turn. In each move the cops either stay where they are, or place one of them on the current position of the robber. The robber must then move from her current position $v$, along an edge, to a successor $w \in v E$ that is not occupied by a cop. If no such position exists, the robber is caught, and the cops have won. Notice that the robber has to leave her current position no matter whether or not a cop has occupied that position. The robber wins if she is never caught, i.e. if the play lasts forever.

Definition 1. The entanglement $\operatorname{ent}(\mathcal{G})$ of a graph $\mathcal{G}$ is the minimal number $k$ such that the cops have a winning strategy in the entanglement game $\mathrm{EG}_{k}(\mathcal{G})$.

The entanglement game is, in essence, a reachability game: the cops try to reach a state of the game at which the robber is captured. It is well known that such games are determined via memoryless strategies, i.e. one of the two players has a winning strategy that depends only on the current position, not on the history of the play. We can therefore restrict our attention to memoryless strategies. 
For a formal definition of strategies in an entanglement game $\mathrm{EG}_{k}(\mathcal{G})$ on a graph $\mathcal{G}=(V, E)$, we describe a play by a sequence $\pi \in S^{\leq \omega}$, where $S=$ $V \times \mathcal{P}_{\leq k}(V)$. Here $\mathcal{P}_{\leq k}(V)$ is the set of subsets of $V$ of size at most $k$, and $(v, P) \in S$ denotes a position where the robber is on $v$ and the cops occupy the vertices in $P$. For brevity, we suppress the information about whose turn to move it is.

A (memoryless) strategy of the robber in $\operatorname{EG}_{k}(\mathcal{G})$ can be described by a partial function $\rho: S \cup\{\epsilon\} \rightarrow V$ with the property that $\rho(v, P) \in v E \backslash P$. Here $\rho(\epsilon)$ describes the choice of the initial vertex by the robber.

Similarly, a (memoryless) strategy of the cops is described by a partial function $\sigma: S \rightarrow V \cup\{\square, \perp\}$ describing which cop, if any, moves to the current vertex occupied by the robber:

- if $\sigma(v, P)=\perp$ then the cops stay where they are, and the next position is $(v, P)$ (but now it is the robber's turn);

- if $\sigma(v, P)=\square$ then it must be the case that $|P|<k$ and the next position is $(v, P \cup\{v\})$ (a cop from outside comes to vertex $v$ );

- otherwise $\sigma(v, P)=u \in P$ (the cop from vertex $u$ goes to $v$ ), and the next position is $(v,(P \backslash\{u\}) \cup\{v\})$.

A strategy $\rho$ of the robber and a strategy $\sigma$ of the cops define a unique play $\pi=\left(v_{0}, P_{0}\right)\left(v_{1}, P_{1}\right)\left(v_{2}, P_{2}\right) \ldots$ that is consistent with $\rho$ and $\sigma$. It starts in position $\left(v_{0}, P_{0}\right)=(\epsilon, \emptyset)$ meaning that the cops and the robber are outside of the graph. After the initial move of the robber the position is $\left(v_{1}, P_{1}\right)=(\rho(\epsilon), \emptyset)$. For every $n>0$ the vertex $v_{2 n+1}$ occupied by the robber after her $(n+1)$-th move is determined by $\rho\left(v_{2 n}, P_{2 n}\right)$, and the set $P_{2 n}$ occupied by the cops after their $n$th move is determined by $\sigma\left(v_{2 n-1}, P_{2 n-1}\right)$. Finally, we have $P_{2 n+1}=P_{2 n}$ and $v_{2 n}=v_{2 n-1}$. A play ends, and is won by the cops, if, for some $n$, there is no position $w \in v_{2 n} E \backslash P_{2 n}$. Infinite plays are won by the robber.

We call a play admissible if there exist memoryless strategies for the robber and the cops such that the play is consistent with them. A robber (or cop) strategy is winning if the robber (cop) wins every (admissible) play consistent with it. Note that we distinguish between cops only according to their position in the graph; in particular, we do not distinguish cops that stay outside of it.

Lemma 2. For every graph $\mathcal{G}$ and every $k$, the game $\mathrm{EG}_{k}(\mathcal{G})$ is determined by memoryless winning strategies, i.e. either the cops or the robber have a winning strategy of the form defined above.

It is easy to characterise the graphs of entanglement zero and one [3].

Proposition 3. Let $\mathcal{G}$ be any finite directed graph.

(1) $\operatorname{ent}(\mathcal{G})=0$ if, and only if, $\mathcal{G}$ is acyclic.

(2) $\operatorname{ent}(\mathcal{G})=1$ if, and only if, $\mathcal{G}$ is not acyclic, and in every strongly connected component there is a node whose removal makes the component acyclic. 
As a consequence, for $k=0$ and $k=1$, the problem whether a given graph has entanglement $k$ is NLOGSPACE-complete.

However, already the graphs of entanglement two provide a quite rich and challenging class.

For the case of undirected graphs, a characterisation of entanglement two has been given by Belkhir and Santocanale [1]. It says that every undirected graph $\mathcal{G}=(V, E)$ of entanglement at most two can be obtained from a forest $\mathcal{T}$ by adding, for every edge $\{a, b\}$ of the forest, new vertices $v_{1}^{a, b}, \ldots, v_{m}^{a, b}$ with edges $\left\{a, v_{i}^{a, b}\right\}$ and $\left\{b, v_{i}^{a, b}\right\}$, for every $i \in\{1, \ldots, m\}$, and possibly deleting the edge $\{a, b\}$.

However, the real interest of entanglement is about directed graphs rather than undirected ones. We generalise the result of Belkhir and Santocanale to directed graphs of entanglement two, and we present two structural characterisations and a kind of a tree decomposition of members of this class.

\section{Graphs of Entanglement Two}

To motivate and give intuition for the class of graphs of entanglement two, we introduce a class $\mathcal{F}$ of graphs $(V, E, F)$ where $F \subseteq V$ is a set of marked vertices. The class $\mathcal{F}$ is defined inductively, as follows:

(1) The graph consisting of one marked vertex and without edges is in $\mathcal{F}$.

(2) $\mathcal{F}$ is closed under removing edges, i.e. if $(V, E, F) \in \mathcal{F}$ and $E^{\prime} \subseteq E$ then $\left(V, E^{\prime}, F\right) \in \mathcal{F}$.

(3) For $\mathcal{G}_{1}, \mathcal{G}_{2} \in \mathcal{F}$ with marked vertices $F_{1}$ and $F_{2}$, the disjoint union of $\mathcal{G}_{1}$ and $\mathcal{G}_{2}$ with marked $F_{1} \cup F_{2}$ is in $\mathcal{F}$.

(4) For $\mathcal{G}_{1}=\left(V_{1}, E_{1}, F_{1}\right), \mathcal{G}_{2}=\left(V_{2}, E_{2}, F_{2}\right) \in \mathcal{F}$, their marked sequential composition $\mathcal{G}$ is in $\mathcal{F}$, where

$$
\mathcal{G}=\left(V_{1} \cup V_{2}, E_{1} \cup E_{2} \cup F_{1} \times V_{2}, F_{1} \cup F_{2}\right) .
$$

(5) For $\mathcal{G}=(V, E, F) \in \mathcal{F}$, the graph $\mathcal{G}^{\prime}$ with added marked loop is in $\mathcal{F}$, where for a new vertex $v$,

$$
\mathcal{G}^{\prime}=(V \cup\{v\}, E \cup(F \times\{v\}) \cup(\{v\} \times V),\{v\}) .
$$

Notice that the rules (2)-(4) add no cycles and do not increase the entanglement. New cycles are created only in item (5), but only between the marked vertices and a new node, which is the only one marked afterwards.

All graphs in the class $\mathcal{F}$ have entanglement two. Before we explain the meaning of the marked vertices $F$ (in Section 4 ) and present the strategy for the cops in $\mathrm{EG}_{2}(\mathcal{G})$ for graphs $\mathcal{G} \in \mathcal{F}$ (in the proof of Theorem 18), let us describe a few sub-classes of $\mathcal{F}$ and possible uses for graphs of entanglement two.

A sub-class of $\mathcal{F}$ are trees with edges directed to the root and, additionally, any set of back-edges going downwards. More formally, such trees can be described as structures $\mathcal{T}=\left(T, E_{T} \cup E_{\text {back }}\right)$ where $\left(T, E_{T}\right)$ is a tree with edges 
directed to the root and for any back-edge $(w, v) \in E_{\text {back }}$ it must be the case that $w$ is reachable from $v$ in $\left(T, E_{T}\right)$. Such graphs have entanglement at most two. A winning strategy for the cops is to chase the robber with one cop until she goes along a back-edge $(w, v)$. Then she is blocked by this cop in the subtree rooted at $w$. Now the second cop chases the robber until she takes another back-edge, and so on, until she is caught at a leaf.

Another class of graphs included in $\mathcal{F}$ are control-flow graphs for structured programs (that do not use goto). Control flow of such programs can be modelled by using sequential and parallel composition (corresponding to items (3) and (4) in the definition of $\mathcal{F}$ ), and loops with single entry and exit point, which are a special case of item $(5)$ in the definition of $\mathcal{F}$.

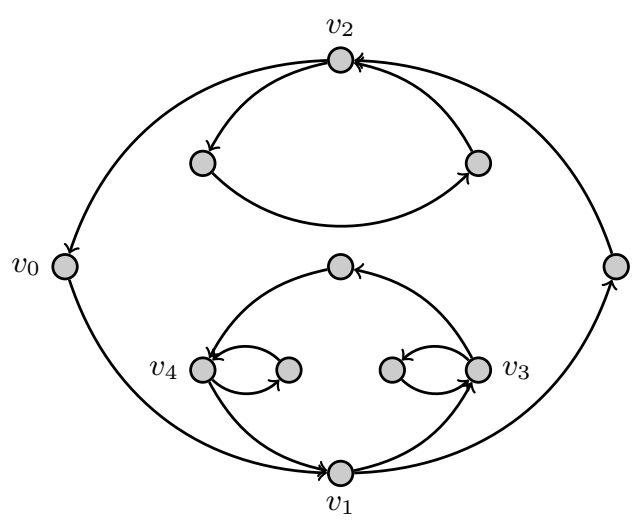

Fig. 1. Example graph of entanglement two.

Consider for example the graph presented in Figure 1. Removing $v_{0}$ from this graph leaves only two non-trivial strongly connected components, namely the $v_{1}$-loop and the $v_{2}$-loop, and one trivial component consisting of a single vertex. ${ }^{2}$ The loops can be decomposed as well by removing $v_{1}$ and $v_{2}$, respectively, and finally the $v_{3}$-loop and the $v_{4}$-loop can be decomposed. This decomposition induces a strategy for the cops, who first place one of them on $v_{0}$ and then chase the robber on $v_{1}$ with the other cop. If the robber enters the $v_{1}$-loop, the cop from $v_{0}$ is used to chase him on $v_{3}$ and $v_{4}$ and so the robber is caught. If the robber does not enter the $v_{1}$-loop, the cop from $v_{1}$ chases him on $v_{2}$ and so the robber is caught.

As one of our main results, we show in Theorem 17 that a decomposition, generalising the above example, can be found for each graph of entanglement two. As a consequence, we prove in Theorem 18 that graphs of entanglement

\footnotetext{
${ }^{2}$ We consider only non-trivial strongly connected components, i.e. not single vertices without self-loops.
} 
two can be characterised in a way similar to the above definition of the class $\mathcal{F}$. More precisely, a graph has entanglement at most two if, and only if, each of its strongly connected components belongs to a class $\mathcal{F}^{\prime}$, which is defined similarly to the class $\mathcal{F}$, but with item (5) changed as follows.

$\left(5^{\prime}\right)$ For $\mathcal{G}=(V, E, F) \in \mathcal{F}^{\prime}$, the graph $\mathcal{G}^{\prime}$ with added loop is in $\mathcal{F}^{\prime}$, where

$$
\mathcal{G}^{\prime}=\left(V \cup\{v\}, E \cup(F \times\{v\}) \cup(\{v\} \times V),\{v\} \cup F^{\prime}\right),
$$

and $F^{\prime}$ is any subset of the previously marked vertices $F$ such that $\mathcal{G}\left[F^{\prime}\right]$ is acyclic and no vertices in $F^{\prime}$ are reachable from $V \backslash F^{\prime}$.

A consequence of our proofs, stated in Proposition 23, is that graphs of entanglement two have both DAG-width and Kelly-width at most 3 . This confirms that graphs of entanglement two are simple according to all known graph measures, and strengthens our motivation to study them as the most basic class of graphs where cycles are already nested in interesting ways.

\section{Entanglement of Graphs with Exit Vertices}

In this section, we introduce a technical notion: the entanglement of a graph with exit vertices, which is crucial for subsequent proofs. To provide intuition for this notion, consider the graph in Figure 1 with the vertex $v_{0}$ removed. This graph contains two non-trivial strongly connected components: the $v_{1}$-loop and the $v_{2}$-loop. The $v_{2}$-loop has entanglement one, so it is clearly simpler than the entire graph. On the other hand, the $v_{1}$-loop has entanglement two. Nevertheless, we claim that also the $v_{1}$-loop is in a sense simpler than the entire graph, despite having the same entanglement. Indeed, observe that not only can two cops capture the robber on the $v_{1}$-loop, but they can do it in such a way that the only vertex through which the robber can exit this loop, $v_{1}$, remains blocked during the whole play after the robber visits it. This observation leads to the notion we study here.

\subsection{Simple and Complex Components}

In the rest of this section, we focus on strongly connected subgraphs of a graph. Let $\mathcal{G}$ be a graph and $\mathcal{G}^{\prime}$ a strongly connected subgraph of $\mathcal{G}$. The set $\operatorname{Ex}\left(\mathcal{G}, \mathcal{G}^{\prime}\right)$ of exit vertices of $\mathcal{G}^{\prime}$ in $\mathcal{G}$ is the set of all $v \in \mathcal{G}^{\prime}$ for which there is a vertex $u \in \mathcal{G} \backslash \mathcal{G}^{\prime}$ with $(v, u) \in E$ (note that we sometimes write $v \in \mathcal{G}$ if $\mathcal{G}=(V, E)$ and $v \in V$ ).

To study subgraphs that contain exit vertices in a way that is independent of the bigger graph in the context, we say that $\mathcal{G}^{*}$ is a graph with exit vertices when $\mathcal{G}^{*}=(V, E, F)$, where $(V, E)$ is a graph and $F$ is any subset of $V$ representing the exits. The following notion is used while decomposing a graph $\mathcal{G}$.

Definition 4. Let $\mathcal{G}$ be a graph and let $v \in \mathcal{G} . A$-component of $\mathcal{G}$ is a graph $\mathcal{C}=(C, E, F)$ with exit vertices such that $(C, E)$ is a strongly connected component of $\mathcal{G} \backslash v$ and $F=\operatorname{Ex}(\mathcal{G}, \mathcal{C})$. 
In a strongly connected graph $\mathcal{G}$, for a vertex $v$, let $\leq_{v}$ be the topological order on the set of strongly connected components of $\mathcal{G} \backslash v$, i.e.

$$
\mathcal{C} \leq{ }_{v} \mathcal{C}^{\prime} \Longleftrightarrow \text { there is a path from } \mathcal{C} \text { to } \mathcal{C}^{\prime} \text { in } \mathcal{G} \backslash v \text {. }
$$

The entanglement game with exit vertices $\mathrm{EG}_{k}^{*}(\mathcal{G})$ is played on a graph $\mathcal{G}=$ $(V, E, F)$ with exit vertices in the same way as the entanglement game, but with an additional winning condition for the robber: she wins a play, when she succeeds in reaching an exit vertex after the last cop has entered $\mathcal{G}$ from outside. More formally, the robber wins a play if it reaches a position $(v, P)$ such that $v \in F$ and $|P|=k$. (This includes the case when the robber already sits on an exit vertex at the time when the last cop comes to that vertex.) In the context of subgraphs inside a larger graph this new winning condition means that the robber can leave the subgraph and get back to the bigger graph.

We define a further variant of the entanglement game to mark the vertex from that a play starts. Let $v$ be a vertex of $\mathcal{G}$. The game $\operatorname{EG}_{k}^{*}(\mathcal{G}, v)$ is played in the same way as $\operatorname{EG}_{k}^{*}(\mathcal{G})$, except that the robber does not choose a vertex to start on, but starts on $v$.

Definition 5. A graph with exit vertices $\mathcal{G}$ is $k$-complex if the robber has a winning strategy (which we call a robber $\mathcal{G}$-strategy) in the entanglement game with exit vertices $\mathrm{EG}_{k+1}^{*}(\mathcal{G})$. If the cops have a winning strategy in $E G_{k+1}^{*}(\mathcal{G})$ (called a cops $\mathcal{G}$-strategy), then $\mathcal{G}$ is $k$-simple.

To start with, let us show that existence of a vertex with only $k$-simple components gives a bound on entanglement.

Proposition 6. If there is a vertex $v$ in a graph $\mathcal{G}$ such that all $v$-components are $k$-simple, then $\operatorname{ent}(\mathcal{G}) \leq k+1$.

Proof. Let $v$ be a vertex such that all $v$-components of $\mathcal{G}$ are $k$-simple. Let $\sigma$ be any strategy for the cops in $\mathrm{EG}_{k+1}(\mathcal{G})$ with the following properties:

- if the robber is on $v$ then chase her there with any cop, i.e. $\sigma(v, P) \neq \perp$,

- if the robber is on a vertex $u$ that is not in a $v$-component, then wait: $\sigma(u, P)=\perp$,

- if the robber is on a vertex $u$ in a $k$-simple $v$-component $\mathcal{C}$, then use a $\mathcal{C}$ strategy $\sigma^{\mathcal{C}}$ moving the cop from $v$ only as the last resort, i.e.

$$
\sigma(u, P)= \begin{cases}\sigma^{C}(u, P \cap \mathcal{C}) & \text { if } \sigma^{C}(u, P \cap \mathcal{C}) \in \mathcal{C} \text { or } \sigma^{C}(u, P \cap \mathcal{C})=\perp, \\ \square & \text { if } \sigma^{C}(u, P \cap \mathcal{C})=\square \text { and } P \backslash \mathcal{C}=\{v\} \text { and }|P| \leq k, \\ w & \text { if } \sigma^{C}(u, P \cap \mathcal{C})=\square \text { and } w \in P \backslash \mathcal{C} \text { with } w \neq v, \\ v & \text { if } \sigma^{C}(u, P \cap \mathcal{C})=\square \text { and }|P|=k+1 .\end{cases}
$$

We show that $\sigma$ is winning for the cops in $\mathrm{EG}_{k+1}(\mathcal{G})$. Assume that the robber has a counter-strategy $\rho$ to win the play that is consistent with both $\rho$ and $\sigma$. First we show that this play visits $v$. Indeed, if it starts in a vertex $v_{0} \neq v$ then 
the robber will either be captured in the $v$-component $\mathcal{C}$ containing $v_{0}$ (we can assume that $v_{0}$ is in a $v$-component, otherwise the cops stay idle until the robber enters such a component or visits $v$ ), or she will be expelled from $\mathcal{C}$, because the cops use a $\mathcal{C}$-strategy. Since we assumed that the robber wins, she is expelled from $\mathcal{C}$. This will continue until $v$ is reached. In this moment an arbitrary cop goes to $v$. Afterwards the cop from $v$ is moved only as the $(k+1)$-st one to enter a component $\mathcal{C}$. Therefore the robber will always either be captured in $\mathcal{C}$ or expelled again without using the cop from $v$ - and thus finally captured.

In the rest of this section, we prove that the converse holds for the case $k=1$. This will lead to Theorem 14 and form the basis of a structural characterisation of graphs of entanglement two in Section 5 .

\subsection{Independence from the Starting Vertex}

Lemma 7. Let $\mathcal{G}$ be a strongly connected $k$-complex graph with exit vertices. Then the robber wins $\mathrm{EG}_{k+1}^{*}(\mathcal{G}, v)$ for all $v \in \mathcal{G}$.

Proof. Let us divide the vertices of $\mathcal{G}$ into two subsets: the set $V_{R}$ of vertices $v$ from which the robber wins $\mathrm{EG}_{k+1}^{*}(\mathcal{G}, v)$ and the set $V_{C}$ of vertices $v$ from which the cops win $\operatorname{EG}_{k+1}^{*}(\mathcal{G}, v)$. These sets are disjoint and as $\mathcal{G}$ is $k$-complex, $V_{R}$ is not empty.

Let us assume that $V_{C}$ is not empty. As $\mathcal{G}$ is strongly connected, there exists an edge from $V_{C}$ to $V_{R}$. Pick such an edge $(w, v) \in E$ and let

- $\rho^{v}$ be a winning strategy for the robber in $\mathrm{EG}_{k+1}^{*}(\mathcal{G}, v)$,

$-\sigma^{w}$ be a winning strategy for the cops in $\operatorname{EG}_{k+1}^{*}(\mathcal{G}, w)$.

First observe that in no play consistent with $\rho^{v}$ does the robber enter $w$ before the last $(k+1)$-st cop moves into $\mathcal{G}$. Indeed, if this was the case, the cops could just continue playing $\sigma^{w}$ from $w$ as if all cops placed already were outside. As $\sigma^{w}$ is winning, this continued play has to end in a position where the robber can neither move nor reach an exit vertex. But this contradicts the fact that the play was consistent with $\rho^{v}$, which is winning for the robber.

We show that the strategy $\rho^{w}$ for the robber which first moves from $w$ to $v$ and then continues playing $\rho^{v}$, ignoring a cop on $w$ if one is placed there in the first move of the cops, is winning. Indeed, if the cops are idle in the first move, then the play is played according to $\rho^{v}$ and thus winning for the robber. In the other case, the play is played according to $\rho^{v}$ as if there was no cop on $w$. But, as observed above, this infinite play never visits $w$ and thus the cop standing there makes no difference - the play is won by the robber.

Since $\rho^{w}$ is winning for the robber in $\operatorname{EG}_{k+1}^{*}(\mathcal{G}, w)$ and $\sigma^{w}$ is winning for the cops in the same game, we get a contradiction. Thus $V_{C}$ is empty, so all vertices of $\mathcal{G}$ belong to $V_{R}$.

The following is a direct corollary (taking $F=\emptyset$ ) of the above lemma. 
Corollary 8 ([4]). Let $\mathcal{G}$ be a strongly connected graph of entanglement $k$. Then the robber wins $\mathrm{EG}_{k}(\mathcal{G})$ with the following change of rules: at the beginning of a play it is not the robber, but the cops who choose the vertex from which the robber has to start.

To prove a converse of Proposition 6 we need to consider various configurations of complex components. We will show that the existence of certain combinations of 1-complex components implies that the graph has entanglement greater than two. This will be used in the Section 4.6 to show that every graph of entanglement two contains a vertex so that after its removal all components are 1-simple. We will later prove that the corresponding property fails for graphs of entanglement $k \geq 3$.

\subsection{Topologically Incomparable Components}

Lemma 9. Let $\mathcal{G}$ be a strongly connected graph and let $v \in \mathcal{G}$. Further, let $\mathcal{C}_{0}$ and $\mathcal{C}_{1}$ be two $k$-complex $v$-components. If $\mathcal{C}_{0}$ and $\mathcal{C}_{1}$ are incomparable with respect to $\leq_{v}$, then $\operatorname{ent}(\mathcal{G})>k+1$.

Proof. Assume that $\mathcal{C}_{0}$ and $\mathcal{C}_{1}$ have entanglement at most $k+1$. Otherwise the robber wins playing in the component of entanglement greater than $k+1$. Thus, the robber's $\mathcal{C}_{0}$-strategy $\rho^{0}$ and the robber's $\mathcal{C}_{1}$-strategy $\rho^{1}$ prescribe the robber to go to an exit vertex when all $k+1$ cops arrive in the component. Note that these strategies are not defined for positions $(w, P)$ where, for some $i \in\{0,1\}$, $w \in \operatorname{Ex}\left(\mathcal{G}, \mathcal{C}_{i}\right)$ and $\left|P \cap \mathcal{C}_{i}\right|=k+1$.

Let $i \in\{0,1\}$. The following strategy $\rho$ is winning for the robber in $\operatorname{EG}_{k+1}(\mathcal{G})$ :

- the robber starts on any vertex $w \in \mathcal{C}_{0}$;

- $\rho(w, P)=\rho^{i}(w, P)$ if $w \in \mathcal{C}_{i},\left|P \cap \mathcal{C}_{i}\right| \leq k+1$, and $\left|P \cap \mathcal{C}_{i}\right|<k+1$ or $w \notin \operatorname{Ex}\left(\mathcal{G}, \mathcal{C}_{i}\right)$

- $\rho(w, P)$ prescribes to run to $\mathcal{C}_{1-i}$ in any possible way if $w \in \operatorname{Ex}\left(\mathcal{G}, \mathcal{C}_{i}\right)$ and $\left|P \cap \mathcal{C}_{i}\right|=k+1$

- $\rho(w, P)$ prescribes to run to $\mathcal{C}_{1-i}$ in any possible way if $w \notin \mathcal{C}_{0} \cup \mathcal{C}_{1}$ and $P \cap \mathcal{C}_{i} \neq \emptyset$.

These cases include all possible positions in a play consistent with $\rho$, because in no play consistent with $\rho$ both $P \cap \mathcal{C}_{0} \neq \emptyset$ and $P \cap \mathcal{C}_{1} \neq \emptyset$ can occur.

To see that $\rho$ is indeed winning for the robber, and that there always is a possible path from $w$ to $\mathcal{C}_{i}$ in the last two cases of the definition above, let us consider a play consistent with $\rho$.

The robber starts on some vertex in $\mathcal{C}_{0}$ and plays $\rho^{0}$ until all $k+1$ cops are in $\mathcal{C}_{0}$. When the last cop moves to $\mathcal{C}_{0}$, she reaches an exit vertex $u$, because $\mathcal{C}_{0}$ is $(k+1)$-complex and $\rho^{0}$ was a $\mathcal{C}_{0}$-strategy. From $u$, she can run to $v$ and then to $\mathcal{C}_{1}$ (without entering $\mathcal{C}_{0}$ again), because the components are incomparable and all paths between them lead through $v$ (note that $v \notin \mathcal{C}_{0}$ and the graph is strongly connected). Now she plays according to $\rho^{1}$ until all $k+1$ cops come to $\mathcal{C}_{1}$, and analogously proceeds to $\mathcal{C}_{0}$ via $v$. This goes on indefinitely, so $k+1$ cops never capture her. 


\subsection{Disjoint Components}

We first consider the case of disjoint components that contain each others basis vertex, and then a more general case.

Lemma 10. Let $\mathcal{G}$ be a strongly connected graph, and let $a_{0}, a_{1} \in \mathcal{G}$ such that, for $i \in\{0,1\}, a_{i}$ is in a $k$-complex $\left(a_{1-i}\right)$-component $\mathcal{C}_{1-i}$. If $\mathcal{C}_{0} \cap \mathcal{C}_{1}=\emptyset$, then $\operatorname{ent}(\mathcal{G})>k+1$.

Proof. The proof of this lemma is analogous to the proof of Lemma 9. Assume again that $\mathcal{C}_{0}$ and $\mathcal{C}_{1}$ have entanglement at most $k+1$. Otherwise the robber wins playing in the component of entanglement greater than $k+1$. Thus, $\mathcal{C}_{0}$-strategy $\rho_{0}$ and $\mathcal{C}_{1}$-strategy $\rho_{1}$ prescribe the robber to go to an exit vertex when all $k+1$ cops arrive in the component.

Let $i \in\{0,1\}$. The following strategy $\rho$ is winning for the robber in $\operatorname{EG}_{k+1}(\mathcal{G})$ :

- the robber starts on any vertex $w \in \mathcal{C}_{0}$;

$-\rho(w, P)=\rho^{i}(w, P)$ if $w \in \mathcal{C}_{i},\left|P \cap \mathcal{C}_{i}\right| \leq k+1$, and $\left|P \cap \mathcal{C}_{i}\right|<k+1$ or $w \notin \operatorname{Ex}\left(\mathcal{G}, \mathcal{C}_{i}\right)$;

- $\rho(w, P)$ prescribes to run along any path leading to $a_{i} \in \mathcal{C}_{1-i}$ until the robber enters $\mathcal{C}_{1-i}$ if $w \in \operatorname{Ex}\left(\mathcal{G}, \mathcal{C}_{i}\right)$ and $\left|P \cap \mathcal{C}_{i}\right|=k+1$;

- $\rho(w, P)$ prescribes to run along any path leading to $a_{i} \in \mathcal{C}_{1-i}$ until the robber enters $\mathcal{C}_{1-i}$ if $w \notin \mathcal{C}_{0} \cup \mathcal{C}_{1}$ and $\left|P \cap \mathcal{C}_{i}\right| \neq \emptyset$.

These cases include all possible positions in a play consistent with $\rho$, because in no play consistent with $\rho$ both $P \cap \mathcal{C}_{0} \neq \emptyset$ and $P \cap \mathcal{C}_{1} \neq \emptyset$ can occur.

To see that $\rho$ is indeed winning for the robber, and that there always is a possible path from $w$ to $\mathcal{C}_{i}$ in the last two cases of the definition above, let us consider a play consistent with $\rho$.

The robber starts on some vertex in $\mathcal{C}_{0}$ and plays $\rho^{0}$ until all $k+1$ cops are in $\mathcal{C}_{0}$. When the last cop moves to $\mathcal{C}_{0}$, she reaches an exit vertex $u$, because $\mathcal{C}_{0}$ is $(k+1)$-complex and $\rho^{0}$ was a $\mathcal{C}_{0}$-strategy. From $u$, she can run to $a_{0}$ and thus (as $a_{0} \in \mathcal{C}_{1}$ ) to $\mathcal{C}_{1}$ (without entering $\mathcal{C}_{0}$ again), because $a_{0} \notin \mathcal{C}_{0}$ and the graph is strongly connected. Now she plays according to $\rho^{1}$ until all $k+1$ cops come to $\mathcal{C}_{1}$, and analogously proceeds to $\mathcal{C}_{0}$ on a way to $a_{1}$. This goes on indefinitely, so $k+1$ cops never capture her.

Lemma 11. Let $\mathcal{G}$ be a strongly connected graph. For $i \in\{0,1\}$, let $\mathcal{C}_{i}$ be two $k$-complex $a_{i}$-components. Let $\mathcal{C}_{0}$ be maximal with respect to $\leq_{a_{0}}$ and let $a_{1} \in \mathcal{C}_{0}$. If $\mathcal{C}_{0} \cap \mathcal{C}_{1}=\emptyset$, then $\operatorname{ent}(\mathcal{G})>k+1$.

Proof. It suffices to prove that $a_{0} \in \mathcal{C}_{1}$, in such case Lemma 10 implies the desired result. Assume, that $a_{0} \notin \mathcal{C}_{1}$. There are tree cases how $\mathcal{C}_{1}$ can be combined with $k$-complex $a_{0}$-components.

Case 1. There is a $k$-complex $a_{0}$-component $\mathcal{C}^{\prime}$ and $\mathcal{C}_{1} \subseteq \mathcal{C}^{\prime}$.

If the components $\mathcal{C}^{\prime}$ and $\mathcal{C}_{0}$ are incomparable with respect to $\leq_{a_{0}}$ then Lemma 9 guarantees a winning strategy for the robber in the entanglement game on $\mathcal{G}$ 
against $k+1$ cops. Because $\mathcal{C}_{0}$ is maximal, we have that $\mathcal{C}^{\prime} \leq_{a_{0}} \mathcal{C}_{0}$ and there is a path $\mathcal{P}_{1}$ from $\mathcal{C}_{1}$ to $\mathcal{C}_{0}$ with $a_{0} \notin \mathcal{P}_{1}$ (see Figure 2).

There is a path $\mathcal{P}_{2}$ from $a_{0}$ to $\mathcal{C}_{1}$, since $\mathcal{G}$ is strongly connected, but no such path includes vertices of $\mathcal{C}_{0}$. Otherwise $\mathcal{C}_{0}$ and $\mathcal{C}^{\prime}$ would be in the same strongly connected component of $\mathcal{G} \backslash a_{0}$. Further, every path $\mathcal{P}_{3}$ from $\mathcal{C}^{\prime}$ to $a_{0}$ (there is at least one) goes through $a_{1}$ (otherwise $a_{0} \in \mathcal{C}_{1}$ ).

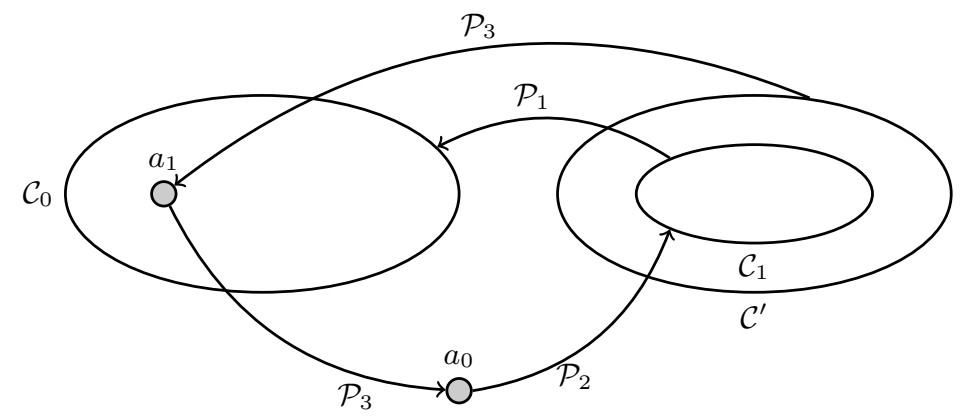

Fig. 2. Case 1: $\mathcal{C}_{1}$ is in an $a_{0}$-component $\mathcal{C}^{\prime}$.

This guarantees that the robber wins the entanglement game on $\mathcal{G}$ against $k+1$ cops switching between $\mathcal{C}^{\prime}$ and $\mathcal{C}_{0}$, because playing according to a $\mathcal{C}_{0^{-}}$ strategy and being expelled from $\mathcal{C}_{0}$ by $k+1$ cops she can reach $a_{0}$ and then $\mathcal{C}_{1}$. Playing according to a $\mathcal{C}_{1}$-strategy and being expelled from $\mathcal{C}_{1}$ she can reach $a_{0}$ and thus $\mathcal{C}_{0}$, which on the way to $a_{0}$. Lemma 7 assures that it makes no difference at which vertex the robber enters $\mathcal{C}^{\prime}$ (or $\mathcal{C}_{0}$ ): she always has a $\mathcal{C}^{\prime}$-strategy (or a $\mathcal{C}_{0}$-strategy).

Case 2. The component $\mathcal{C}_{1}$ includes vertices of two different strongly connected components of $\mathcal{G} \backslash a_{0}$.

Then there is a path in $\mathcal{C}_{1}$ from one such strongly connected component to the other that does not go through $a_{1}$, but through $a_{0}$. (If all such paths avoided $a_{0}$, the two strongly connected components would not be distinct.) But then we have $a_{0} \in \mathcal{C}_{1}$.

Case 3. $\mathcal{C}_{1}$ does not include vertices of different $a_{0}$-components and is not a strict subset of a $k$-complex $a_{0}$-component.

Due to our assumption, $a_{0} \notin \mathcal{C}_{1}$, and we distinguish two subcases.

Case $3 a . \mathcal{C}_{1}$ consists of some vertices from an $a_{0}$-component $\mathcal{C}^{\prime}$ and some vertices that are in no strongly connected component of $\mathcal{G} \backslash a_{0}$.

In this case, these vertices must also be a part of $\mathcal{C}^{\prime}$, because all vertices of $\mathcal{C}_{1}$ are connected by paths that contain neither $a_{0}$ nor $a_{1}$. So, in fact, this subcase is not possible.

Case 3b. $\mathcal{C}_{1}$ lies in a $k$-simple $a_{0}$-component $\mathcal{C}^{\prime}$.

We show that because $\mathcal{C}_{1}$ is $k$-complex, $\mathcal{C}^{\prime}$ must be $k$-complex as well, which contradicts the assumption of this subcase. We describe a $\mathcal{C}^{\prime}$-strategy for the 
robber. She starts in $\mathcal{C}_{1}$ and plays according to her $\mathcal{C}_{1}$-strategy. We can assume that it prescribes to wait until all $k+1$ cops come to $\mathcal{C}_{1}$, because otherwise $\operatorname{ent}\left(\mathcal{C}_{1}\right)>k+1$ and $\operatorname{ent}\left(\mathcal{C}^{\prime}\right)>k+1$. When all cops come to $\mathcal{C}_{1}$ the robber can leave $\mathcal{C}_{1}$. We show that she can leave $\mathcal{C}^{\prime}$ as well. It suffices to show that from every $v \in \operatorname{Ex}\left(\mathcal{C}^{\prime}, \mathcal{C}_{1}\right)$ there is a path to a vertex $w \in \operatorname{Ex}\left(\mathcal{G}, \mathcal{C}^{\prime}\right)$ that avoids $\mathcal{C}_{1}$ (except the vertex $v$ ). Otherwise every path $\mathcal{P}$ from $v$ to some $w$ (there is such path because $\mathcal{C}^{\prime}$ is strongly connected) leaves $\mathcal{C}_{1}$, goes through at least one vertex $u \in \mathcal{C}^{\prime} \backslash \mathcal{C}_{1}$ and then goes back to $\mathcal{C}_{1}$. Then $a_{1} \notin \mathcal{P}$ because $\mathcal{P} \subseteq \mathcal{C}^{\prime}, a_{1} \in \mathcal{C}_{0}$, and $\mathcal{C}^{\prime}$ and $\mathcal{C}_{0}$ are distinct $a_{0}$-components. So we have $u \in \mathcal{C}_{1}$, but we assumed that $u \notin \mathcal{C}_{1}$.

We give an example showing that the maximality of $\mathcal{C}_{0}$ in Lemma 11 is essential. Consider the graph in Figure 3. All requirements of Lemma 11 are fulfilled for this graph except the maximality of $\mathcal{C}_{0}: \mathcal{C}_{0}$ is a 1-complex $a_{0}$-component, $\mathcal{C}_{1}$ is a 1-complex $a_{1}$-component, and $a_{1} \in \mathcal{C}_{0}$. The entanglement of the graph is two, although $\mathcal{C}_{0}$ and $\mathcal{C}_{1}$ are disjoint. The cops have the following winning strategy. We only assume moves of the robber that lead to a strongly connected cop free subgraph. The cops expel the robber from $\mathcal{C}_{1}$, if she is there, and place one of the cops on vertex $a_{1}$, which must be visited by the robber leaving $\mathcal{C}_{1}$. The robber visits vertex $v$ and the other cop goes there. The robber proceeds to $w$ and the cop who is not on $v$ occupies $w$. Then the cop from $v$ forces the robber to leave $\mathcal{C}_{1}$ and follows her to $a_{1}$. The robber visits $v$ again, the cop from $a_{1}$ follows her there. As vertex $w$ is occupied, the robber has to remain in $\mathcal{C}_{0} \cup\left\{a_{0}\right\}$. The cop from $w$ goes to $a_{1}$ and captures the robber.

Note that we actually have shown that all $w$-components are 1-simple and used the strategy for the cops described in the proof of Proposition 6.

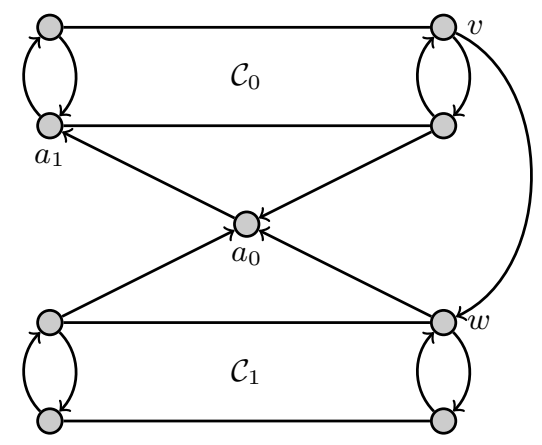

Fig. 3. Importance of maximality of the components.

\subsection{Pairwise Intersecting 1-Complex Components}

Lemma 12. Let $\mathcal{G}$ be a strongly connected graph. Let $I=\{0, \ldots, m\}$ be an index set for some $m \in\{1, \ldots,|V|-1\}$. For $i \in I$, let $a_{i} \in \mathcal{G}$ and let $\mathcal{C}_{i}$ be $a$ 
1-complex $a_{i}$-component such that $a_{i} \in \mathcal{C}_{j}$ for all $i \neq j$ and $j \in I$. If $\bigcap_{i \in I} \mathcal{C}_{i}=\emptyset$, then $\operatorname{ent}(\mathcal{G})>2$.

Proof. If $m=1$, then we have the conditions of Lemma 10, so assume that $m \geq 2$. We, further, can assume that $\operatorname{ent}\left(\mathcal{C}_{i}\right) \leq 2$ for all $i \in I$. Then $\mathcal{C}_{i}$-strategies prescribe the robber to wait in the component until both cops come and then to reach an exit vertex.

We give a winning strategy for the robber in the game $\mathrm{EG}_{2}(\mathcal{G})$. She starts in a cop free component $\mathcal{C}_{j}$ and plays according to her $\mathcal{C}_{j}$-strategy. When the second cop comes to $\mathcal{C}_{j}$ she escapes from $\mathcal{C}_{j}$. Now it suffices to show that she can reach a new cop free component. Let the second cop come to $\mathcal{C}_{j}$ on a vertex $v$, the first cop being on a vertex $w \in \mathcal{C}_{j}$. At this point, since $\bigcap_{l \in I} \mathcal{C}_{l}=\emptyset$, there is an $a_{i}$-component $\mathcal{C}_{i}$ with $w \notin \mathcal{C}_{i}$. If $v \in \mathcal{C}_{i}$, the robber plays her $\mathcal{C}_{i}$-strategy starting from $v$ and assuming that a cop followed her there. If $v \notin \mathcal{C}_{i}$, then the robber can escape from $\mathcal{C}_{j}$ and reach $a_{j}$, which is in the cop free component $C_{i}$. On entering $\mathcal{C}_{i}$, the robber continues with a $\mathcal{C}_{i}$-strategy.

\subsection{A Vertex Having Only Simple Components}

Before we prove the main theorem, we need one more lemma about possible configurations of incomparable strongly connected components.

Lemma 13. Let $\mathcal{G}$ be a strongly connected graph. Let $\mathcal{C}_{v}$ be a $v$-component, and $\mathcal{C}_{w}$ be a w-component of $\mathcal{G}$, for distinct vertices $v$ and $w$ such that $\mathcal{C}_{v} \cap \mathcal{C}_{w} \neq \emptyset$ and $\mathcal{C}_{v} \nsubseteq \mathcal{C}_{w}$. If $v$ is in $\mathcal{C}_{w}$, then $w$ is in $\mathcal{C}_{v}$.

Proof. Assume that the conditions of the lemma hold, but $w \notin \mathcal{C}_{v}$ (Figure 4). Let $u \in \mathcal{C}_{v} \cap \mathcal{C}_{w}$ and $u^{\prime} \in \mathcal{C}_{v} \backslash \mathcal{C}_{w}$. Because $u^{\prime}, u \in \mathcal{C}_{v}$, which is strongly connected, there are paths from $u^{\prime}$ to $u$ and vice versa that do not include $v$. None of these paths includes $w$ (because otherwise $w \in \mathcal{C}_{v}$ ), so $u^{\prime}$ and $u$ lie in the same $w$ component. But we assumed that $u^{\prime} \notin \mathcal{C}_{w}$, and $u \in \mathcal{C}_{w}$, and $C_{w}$ is strongly connected: contradiction.

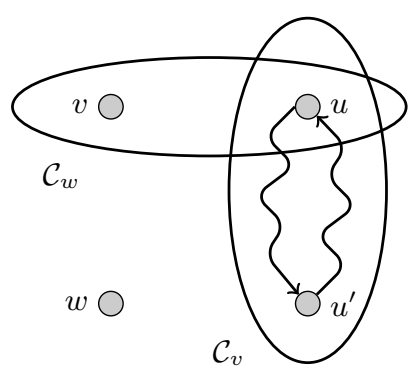

Fig. 4. The $w$-component $\mathcal{C}_{w}$ contains $v$, but the $v$-component $\mathcal{C}_{v}$ does not include $w$.

With the above lemma, we can finally prove the converse of Proposition 6 . 
Theorem 14. On a strongly connected graph $\mathcal{G}=(V, E)$, two cops have a winning strategy in the game $\mathrm{EG}_{2}(\mathcal{G})$ if and only if there exists a vertex $a \in \mathcal{G}$ such that every a-component is 1-simple.

Proof. The direction from right to left is proven in Proposition 6: if every acomponent is 1 -simple, then $\operatorname{ent}(\mathcal{G}) \leq 2$. We show the other direction.

Towards a contradiction, assume that the cops win $\mathrm{EG}_{2}(\mathcal{G})$, but, for all $a \in V$ there is a $a$-component $\mathcal{C}$ of $\mathcal{G}$ such that they lose $\operatorname{EG}_{2}^{*}(\mathcal{C})$.

We construct a sequence $a_{0}, a_{1}, \ldots, a_{m}$ of vertices from $V$ and a sequence $\mathcal{C}_{0}, \mathcal{C}_{1}, \ldots, \mathcal{C}_{m}$ of corresponding $a_{i}$-components $\mathcal{C}_{i}$. We require that all $\mathcal{C}_{i}$ are maximal 1-complex $a_{i}$-components with respect to $\leq_{a_{i}}$, and that $\bigcap_{i=0}^{m} \mathcal{C}_{i} \neq \emptyset$.

Take an arbitrary vertex as $a_{0}$. There is a 1 -complex $a_{0}$-component $\mathcal{C}_{0}$, due to the assumption. Choose among all such strongly connected components a maximal one with respect to $\leq_{a_{0}}$. In general, suppose that $a_{i}$ and $\mathcal{C}_{i}$ are already constructed, and, for $j \leq i$, every $\mathcal{C}_{j}$ is maximal with respect to $\leq_{a_{j}}$, and $\bigcap_{j \leq i} \mathcal{C}_{j} \neq \emptyset$ holds. Choose a vertex $a_{i+1}$ from $\bigcap_{j \leq i} \mathcal{C}_{j}$ and a 1-complex $a_{i+1^{-}}$ component $\mathcal{C}_{i+1}$ that is maximal with respect to $\leq_{a_{i+1}}$. Due to Lemma 11, it intersects all $\mathcal{C}_{j}$, for $j \leq i$ (otherwise $\operatorname{ent}(\mathcal{G})>2$ ). By Lemma $13, a_{i} \in \mathcal{C}_{j}$, for all $i \neq j$. Thus, according to Lemma $12, \bigcap_{j \leq i+1} \mathcal{C}_{j} \neq \emptyset$ (or otherwise $\operatorname{ent}(\mathcal{G})>2$ ), and we can continue the construction.

Note that for all $i, a_{i} \notin \mathcal{C}_{i}$. Finally, for some $m<|V|$, there is no corresponding 1-complex $\left(a_{m+1}\right)$-component for $a_{m+1}$ and the construction stops. This means that all $a_{m+1}$-components are 1-simple, which contradicts our assumption that for every vertex $a$ there is a 1-complex $a$-component. Otherwise there is a 1 -complex $a_{m+1}$ component $\mathcal{C}_{m+1}$, but $\bigcap_{i=0}^{m+1} \mathcal{C}_{i}=\emptyset$. In this case we have $\operatorname{ent}(\mathcal{G})>2$, according to Lemma 12 .

It is clear that the entanglement of a graph is at most two if and only if the entanglement of all its strongly connected components is at most two, so we have the following corollary.

Corollary 15. Let $\mathcal{G}$ be a graph. In $\mathrm{EG}_{2}(\mathcal{G})$, the cops have a winning strategy if and only if in every strongly connected component $\mathcal{C}$ of $\mathcal{G}$, there exists a vertex $a \in \mathcal{C}$, such that every a-component of $\mathcal{C}$ is 1 -simple.

Note that the above fails for graphs of entanglement three or greater, as proven in Section 7.

\section{Decompositions for Entanglement Two}

The proof of Theorem 14 shows the structure of a strongly connected graph $\mathcal{G}$ of entanglement two. It has a vertex $a_{0}$ such that the graph $\mathcal{G} \backslash a_{0}$ can be decomposed in 1-simple $a_{0}$-components. We can divide them into two classes: leaf components, from which one cop expels the robber, and inner components, where one cop does not win, but blocks all exit vertices making the other cop free from guarding the simple component. It turns out that every inner component $\mathcal{C}_{0}$ 
again has a vertex $a_{1}$ such that $\mathcal{C}_{0}$ decomposes in 1-simple $a_{1}$-components an so on. We shall show that $a_{1}$ is the vertex where the second cop stays (blocking all exit vertices of $\mathcal{C}_{0}$ ) when the first cop leaves $a_{0}$. Let us define the decomposition for graphs of entanglement two.

Definition 16. An entanglement two decomposition of a strongly connected graph $\mathcal{G}=\left(V_{G}, E_{G}\right)$ is a triple $(\mathcal{T}, F, g)$, where $\mathcal{T}$ is a nontrivial directed tree $\mathcal{T}=(T, E)$ with root $r$ and edges directed away from the root, and $F$ and $g$ are functions $F: T \rightarrow 2^{V_{G}}$ and $g: T \rightarrow V_{G}$ with the following properties:

(1) $F(r)=V_{G}$

(2) $g(v) \in F(v)$ for all $v \in T$,

(3) if $\left(v, w_{1}\right) \in E$ and $\left(v, w_{2}\right) \in E$, then $F\left(w_{1}\right) \cap F\left(w_{2}\right)=\emptyset$, for $w_{1} \neq w_{2}$,

(4) for $(v, w) \in E, \mathcal{G}[F(w)]$ is a strongly connected component of $\mathcal{G}[F(v)] \backslash g(v)$,

(5) the subgraph of $\mathcal{G}$ induced by the vertex set $(F(v) \backslash g(v)) \backslash\left(\bigcup_{w \in v E} F(w)\right)$ is acyclic for all $v \in T$,

(6) no vertex in $\operatorname{Ex}(\mathcal{G}, \mathcal{G}[F(v)])$ is reachable from $\mathcal{G}\left[\bigcup_{w \in v E} F(w)\right]$ in $\mathcal{G} \backslash g(v)$, for all $v \in T$.

We shall call tree vertices and (abusing the notation) their F-images bags and $g$-images decomposition points.

Note that from the definition follows that if $(v, w) \in E$ then $F(w) \subsetneq F(v)$, and that if $v \in T$ is a leaf in $\mathcal{T}$ then $\mathcal{G}[F(v)] \backslash g(v)$ is acyclic. Observe further that successors of a bag are partially ordered in the sense that, for each bag $v$, its successors $v E=\left\{w_{1}, \ldots, w_{m}\right\}$ form a DAG $\mathcal{D}=\left(v E, E_{D}\right)$ such that, for all $w_{i}, w_{j} \in v E, w_{j}$ is reachable from $w_{i}$ in $\mathcal{D}$ if and only if $F\left(w_{j}\right)$ is reachable from $F\left(w_{i}\right)$ in $\mathcal{G}[F(v)] \backslash g(v)$. An example of a graph and its entanglement two decomposition is given in Figure 5.

We look again at the class of trees with back-edges defined in Section 3. Let us look at decompositions of members of graph classes defined in Section 3. The decomposition tree of a tree with back-edges $\mathcal{T}=\left(T, E_{T}, E_{\text {back }}\right)$ can be given as $\left(T^{\prime}, E_{T}^{\prime}, F, \mathrm{id}_{T^{\prime}}\right)$ where $T^{\prime}$ is $T$ without leaves, $E_{T}^{\prime}$ is

$$
\left\{(v, w) \mid(w, v) \in E_{T} \text { and } v \text { is not a leaf in } \mathcal{T}\right\}
$$

and if $v \in T^{\prime}$ then $F(v)$ is the subtree rooted at $v$ and $g(v)=v$. It is easy to verify that $\left(T^{\prime}, E_{T}^{\prime}, F, \operatorname{id}_{T^{\prime}}\right)$ is an entanglement two decomposition of $\mathcal{T}$.

\subsection{The Main Results}

Having defined the decomposition for entanglement two, we are ready to state our two main results characterising directed graphs of entanglement two.

Theorem 17. A strongly connected graph $\mathcal{G}=(V, E)$ has entanglement at most two if and only if $\mathcal{G}$ has an entanglement two decomposition. 

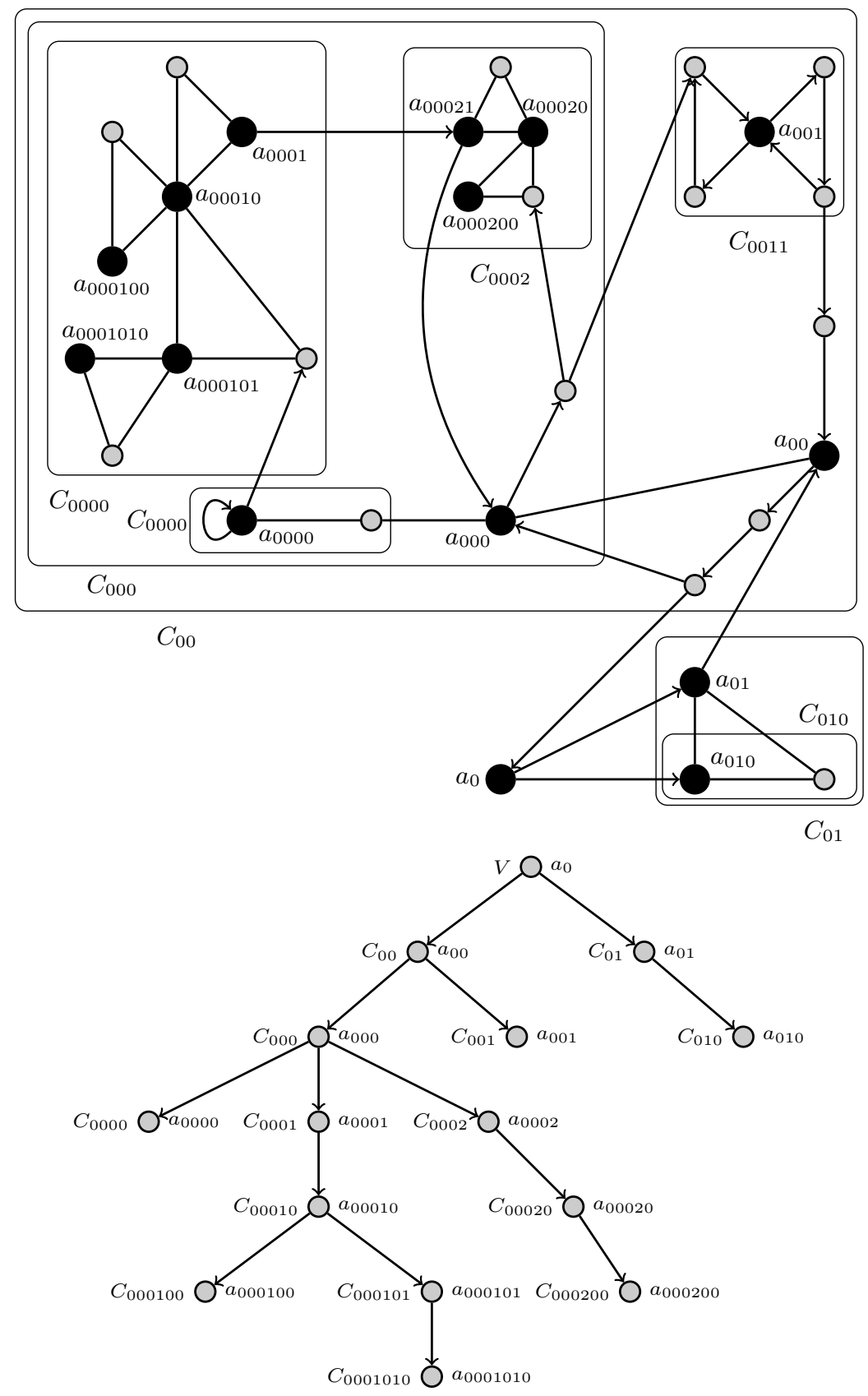

Fig. 5. A typical graph of entanglement two and its entanglement two decomposition. On the upper picture, the components (images of function $F$ ) are shown as squares (only up to level 4), blocking vertices (images of function $g$ ) are shown as filled circles. On the picture below, the decomposition tree of the graph is given. The bags are labelled with images from functions $F$ and $g$. 
The above theorem, which we will prove in the subsequent subsections, allows us to complete the characterisation of directed graphs of entanglement two given in Section 3. Observe first, that there is a connection between the entanglement two decomposition and the characterisations of undirected graphs of entanglement two given by Belkhir and Santocanale [1].

For an entanglement two decomposition of an undirected graph $\mathcal{G}=(V, E)$, consider a connected component, which is an undirected tree $\mathcal{T}=\left(V_{T}, E_{T}\right)$ with additional vertices (as described in Section 2). Choose an arbitrary leaf $v \in V_{T}$ as a root. We get a decomposition tree after orienting all edges from $E_{T}$ (if an edge was deleted, restore it before orienting) away from the root and deleting all leaves other than $v$. We define the functions $F$ and $g$ as follows: $F(v)$ is $V_{T}$ and $g(v)$ is $v$. In general, if, for a bag $w$, the functions $F$ and $g$ on $w$ are already defined, let $\mathcal{C}$ be a strongly connected component of $\mathcal{G}[F(w)] \backslash g(w)$. Choose a vertex $u$ in $\mathcal{C}$ with an edge between $w$ and $u$ and set $F(u)=\mathcal{C}$ and $g(u)=u$.

Recall the definition of the class $\mathcal{F}^{\prime}$ in Section 3 for the following theorem.

Theorem 18. A strongly connected directed graph $\mathcal{G}$ has entanglement at most two if and only if $\mathcal{G} \in \mathcal{F}^{\prime}$.

Proof. Let $\mathcal{G}=(V, E)$ be a strongly connected directed graph of entanglement at most two. We prove that $\mathcal{G}$ can be constructed using operations (1)-(4), ( $\left.5^{\prime}\right)$ from the definition of the class $\mathcal{F}^{\prime}$. Let $\mathcal{T}=\left(T, E_{T}, F, g\right)$ be an entanglement two decomposition of $\mathcal{G}$. We prove by induction on the structure of $\mathcal{T}$ in a bottom-up manner that one can construct all successor bags $F\left(w_{1}\right), \ldots, F\left(w_{m}\right)$ of a bag $v$ such that, for all $i=1, \ldots, m$, the marked vertices of $F\left(w_{i}\right)$ include $g\left(w_{i}\right)$ and all vertices that are not reachable in $\mathcal{G}[F(v)] \backslash g(v)$ from a bag $F\left(w_{i}\right)$.

A leaf bag $F(v)$ becomes acyclic when the vertex $g(v)$ is deleted. First, we construct $\mathcal{G}[F(v)] \backslash g(v)$ such that all vertices are marked, which is possible with the operations $(1)-(4)$. Then we apply rule $\left(5^{\prime}\right)$ adding vertex $g(v)$ such that the whole bag $F(v)$ is marked. This marking is possible as $\mathcal{G}[F(v)] \backslash g(v)$ is acyclic.

Having constructed all bags $F\left(w_{1}\right), \ldots, F\left(w_{m}\right)$ with marked vertices as in the induction hypothesis described above, we construct the bag $F(v)$. Let $v E_{T}=$ $\left\{w_{1}, \ldots, w_{m}\right\}$. Note that $F(v)$ consists of $g(v)$, all bags $F\left(w_{i}\right)$ of the next lower level, and vertices of $F(v) \backslash g(v)$ not reachable from a bag $F\left(w_{i}\right)$ within $\mathcal{G}[F(v)] \backslash$ $g(v)$. We denote the latter vertices by $A$ and the induced subgraph $\mathcal{G}[A]$ by $\mathcal{A}$. Our aim is to construct $\mathcal{G}[F(v)]$ such that marked vertices are precisely $g(v)$ and the vertices of $A$. We first construct $\mathcal{A}$ using rules (1)-(4) such that all vertices of $\mathcal{A}$ are marked. Then we apply rule (3) to get the disjoint union of $\mathcal{A}$ and bags $\mathcal{G}\left[F\left(w_{i}\right)\right]$. If there are edges from $\mathcal{A}$ to a bag $F\left(w_{i}\right)$ we add these with rule (4), which is possible because all vertices in $\mathcal{A}$ are marked. Now we use rule $\left(5^{\prime}\right)$ to add vertex $g(v)$ and the edges (that exist in $\mathcal{G}$ ) between $g(v)$, and $F\left(w_{i}\right)$ and $\mathcal{A}$. We show that this is possible. There can be edges in $\mathcal{G}$ of the following kinds:

- From $\operatorname{Ex}\left(\mathcal{G}, \mathcal{G}\left[F\left(w_{i}\right)\right]\right)$ to $g(v)$. We can add these, as vertices of $\operatorname{Ex}\left(\mathcal{G}, \mathcal{G}\left[F\left(w_{i}\right)\right]\right)$ are not reachable from $\bigcup_{i=1}^{m} F\left(w_{i}\right)$ in $\mathcal{G}[F(v)] \backslash g(v)$ (due to property (6) of the entanglement two decomposition) and thus are contained in $\mathcal{A}$. But $A$ is marked by induction hypothesis. 
- From $\mathcal{A}$ to $g(v)$. We can add these edges because $\mathcal{A}$ is marked.

- From $g(v)$ to any vertex in $F(v)$. This is possible due to rule $\left(5^{\prime}\right)$.

There are no other edges in $\mathcal{G}$ between $g(v), \mathcal{A}$ and $F\left(w_{i}\right)$ because of the definition of $\left.\operatorname{Ex}\left(\mathcal{G}, \mathcal{G}\left[F\left(w_{i}\right)\right]\right)\right)$. It remains to define marked vertices in $F(v)$. Vertex $g(v)$ is marked (rule $\left(5^{\prime}\right)$ ) as needed for induction hypothesis. We also let vertices in $A$ remain marked. (This is needed because these can be exit vertices of $\mathcal{G}[F(v)]$ in $\mathcal{G}$.) Note that $A$ is not reachable from a bag $F\left(w_{i}\right)$ in $\mathcal{G}[F(v)] \backslash g(v)$, so these vertices must be marked as well.

For the other direction, assume that $\mathcal{G}=(V, E)$ is strongly connected and in $\mathcal{F}^{\prime}$. Note that during the construction of $\mathcal{G}$ we get a sequence of graphs with marked vertices. We show by induction on the construction of $\mathcal{G}$ according to rules (1)-(4), $\left(5^{\prime}\right)$ that the cops have a winning strategy in the game $\mathrm{EG}_{2}^{*}(V, E, F)$ where $F$ is the set of marked vertices of $\mathcal{G}$. The graph consisting of one vertex and without edges (arising after the application of rule (1)) has entanglement zero. Applications of rules (2)-(4) do not increase entanglement because they do not introduce new cycles. Assume that two cops have a winning strategy $\sigma$ on a graph $\mathcal{G}^{\prime}=\left(V^{\prime}, E^{\prime}, F^{\prime}\right)$ with marked vertices $F^{\prime}$. Let $\mathcal{G}^{\prime \prime}$ be the graph we get from $\mathcal{G}^{\prime}$ after adding a new vertex $v$ via rule $\left(5^{\prime}\right)$. We give a winning strategy for the cops on $\mathcal{G}^{\prime \prime}$. First, they play according to $\sigma$ on $\mathcal{G}^{\prime}$ thus capturing the robber or expelling her to $v$. When she visits $v$ one cop follows her there. The robber runs to a strongly connected component of $\mathcal{G}^{\prime}$. The cops play again according to $\sigma$ using the other cop (who is not on $v$ ) first and letting the cop on $v$ guard $\mathcal{G}^{\prime}$. When $\sigma$ prescribes to use the second cop in $\mathcal{G}^{\prime}$ the robber cannot escape from $\mathcal{G}^{\prime}$ any more (because $\sigma$ is a winning strategy for the cops in $\mathrm{EG}^{*}\left(\mathcal{G}^{\prime}\right)$ ). So the cops capture the robber in $\mathcal{G}^{\prime}$ and thus also in $\mathcal{G}^{\prime \prime}$.

\subsection{A Characterisation of 1-Complex Components}

Lemma 19. Let $\mathcal{G}=(V, E, F)$ be a strongly connected graph with exit vertices. If, for all $v \in V$, there is a cycle $\mathcal{C}$ in $\mathcal{G} \backslash v$ from that a vertex in $F$ is reachable in $\mathcal{G} \backslash v$, then $\mathcal{G}$ is 1-complex.

Proof. Let $C(v)$ be a cycle in $\mathcal{G} \backslash v$ from which a vertex in $F$ is reachable in $\mathcal{G} \backslash v$. Let $\mathcal{C}$ be any cycle in $\mathcal{G}$. The following strategy $\rho$ is winning for the robber in $\mathrm{EG}_{2}^{*}(\mathcal{G})$.

- start on an arbitrary vertex in $\mathcal{C}$;

- $\rho(v, \emptyset)$ prescribes the robber to stay in $\mathcal{C}$;

- $\rho(v,\{w\})$ prescribes to run to a vertex in the cycle $C(w)$ if $v \notin C(w)$;

- $\rho(v,\{w\})$ prescribes to stay in the cycle $C(w)$ if $v \in C(w)$;

- $\rho(v,\{w, u\})$ prescribes to run to an exit vertex (and thus win).

By the assumption, in a position $(v,\{w, v\})$ there is a cop free path (possibly except the cop on $v$ ) to an exit vertex, so $\rho$ is indeed winning for the robber. 
Let $\mathcal{G}$ be a graph with exit vertices. We call a vertex $v \in \mathcal{G}$ a blocking vertex, if there is no strongly connected component of $\mathcal{G} \backslash v$ from which there is a path to an exit vertex in $\mathcal{G} \backslash v$. We denote the set of blocking vertices $B(\mathcal{G})$ and define a binary relation $\rightarrow$ on $B(\mathcal{G})$ :

$$
v \rightarrow w \Longleftrightarrow w \text { is not on a cycle in } \mathcal{G} \backslash v \text {. }
$$

Lemma 20. If $\mathcal{G}=(V, E, F)$ is a 1-simple graph with exit vertices then the relation $\rightarrow$ on $B(\mathcal{G})$ is a total preorder, i.e. it is transitive and total.

Proof. For transitivity, let $u, v, w \in B(\mathcal{G})$ and assume that it is $u \rightarrow v$ and $v \rightarrow w$. Then all cycles with $w$ contain $v$ and all cycles with $v$ contain $u$. It follows that all cycles with $w$ contain $u$ and $w$ is not on a cycle in $\mathcal{G} \backslash u$.

It remains to show the totality of $\rightarrow$. Because the reflexivity is trivial, let $v$ and $w$ be distinct vertices in $B(\mathcal{G})$. Assume that neither $v \rightarrow w$ nor $w \rightarrow v$ holds, i.e. $w$ is on a cycle $\mathcal{C}_{v}$ in $\mathcal{C} \backslash v$ and $v$ is on a cycle $\mathcal{C}_{w}$ in $\mathcal{C} \backslash w$. Further, every path from $\mathcal{C}_{v}$ to an exit vertex leads through $v$, because $v$ is blocking, and there is such a path, because $\mathcal{G}$ is strongly connected. Consider the part of this path from $v$ to an exit vertex. Together with $\mathcal{C}_{w}$ it witnesses that $w$ is not blocking, in contradiction to the choice of $w$.

Note that $\rightarrow$ is not necessarily antisymmetric, so we define the symmetrisation $\sim$ of $\rightarrow$ on $B(\mathcal{G})$ and extend the relation $\rightarrow$ on $B(\mathcal{G}) / \sim$. Let $[v]$ denote the equivalence class of $v$ with respect to $\sim$. The binary relation $\rightarrow \sim$ is well defined by

$$
[v] \rightarrow \sim[w] \Longleftrightarrow v \rightarrow w .
$$

The transitivity and the totality are inherited by $\rightarrow \sim$ from $\rightarrow$, the antisymmetry is guaranteed by including all not antisymmetric pairs of elements into the same class, thus the following holds.

Lemma 21. If $\mathcal{G}$ is a 1-simple graph with exit vertices then the relation $\rightarrow \sim$ on $B(\mathcal{G})$ is a total order.

If, for vertices $v$ and $w$ in a graph with exit vertices $\mathcal{G}, v \rightarrow w$ holds then we say that vertex $v$ blocks vertex $w$. The next lemma follows from the previous one.

Lemma 22. If $\mathcal{G}=(V, E, F)$ is a 1-simple graph with exit vertices such that $(V, E)$ has entanglement two then there is a vertex $v \in \mathcal{G}$ that blocks all vertices from $B(\mathcal{G})$.

\subsection{The Correctness of the Decomposition}

Theorem 17 A strongly connected graph $\mathcal{G}=(V, E)$ has entanglement at most two if and only if $\mathcal{G}$ has an entanglement two decomposition. 
Proof.

$(\Rightarrow)$ For a graph $\mathcal{G}$ with ent $(\mathcal{G})=2$, we construct the tree $\mathcal{T}=\left(T, E_{T}\right)$ and the functions $F$ and $g$ in a top-down manner. In each step we enlarge the tree adding to a bag $v$ that is currently a leaf some successors $\left\{w_{1}, \ldots, w_{m}\right\}$ and define the functions $F$ and $g$ on them. We require that all $g\left(w_{i}\right)$-components of $\mathcal{G}\left[F\left(w_{i}\right)\right]$ are 1-simple.

To start with, by Theorem 14 there exists a vertex $a_{0} \in V$ such that all $a_{0^{-}}$ components of $\mathcal{G}$ are 1-simple. For the root $r$ of the tree $\mathcal{T}$ we set $F(r)=V$ and $g(r)=a_{0}$. In general, for every bag $v$ that is a leaf of the already constructed part of the tree, let $C_{1}, \ldots, C_{m}$ induce all strongly connected components of $F(v) \backslash g(v)$. If there are no such components (i.e. $m=0$ ), skip this bag and proceed with a next one, if there is any. If $m \geq 1$, create, for each $i \in\{1, \ldots, m\}$, a successor $w_{i}$ of $v$ and set $F\left(w_{i}\right)=C_{i}$. From the construction we know that each $C_{i}$ induces a 1-simple $g(v)$-component. If it has a vertex $a$ whose removal makes the component acyclic, i.e. the cops win $\operatorname{EG}_{1}\left(\mathcal{G}\left[C_{i}\right]\right)$, then set $g\left(w_{i}\right)=a$. If the cops lose $\mathrm{EG}_{1}\left(\mathcal{G}\left[C_{i}\right]\right)$ then, according to the definition of a 1-simple component, one cop can block all exit vertices (to win with help of the other cop), i.e. he can place himself on a blocking vertex of $\mathcal{G}\left[C_{i}\right]$. Among all blocking vertices there is a vertex $a$ that blocks all vertices in $B\left(\mathcal{G}\left[C_{i}\right]\right)$, due to Lemma 22 . Set $g(v)=a$. Then all $a$-components of $\mathcal{G}\left[F\left(w_{i}\right)\right]$ are 1-simple. We check that all requirements of the entanglement two decomposition are fulfilled. The first four properties follow immediately from the construction. Let $v E_{T}=\left\{w_{1}, \ldots, w_{m}\right\}$. Then the subgraph of $\mathcal{G}$ induced by the vertex set $(F(v) \backslash g(v)) \backslash\left(\bigcup_{i=1}^{m} F\left(w_{i}\right)\right)$ is acyclic because a cycle would induce a new strongly connected component, but $\bigcup_{i=1}^{m} F\left(w_{i}\right)$ includes all components of $F(v)$. Finally assume that a vertex $w \in$ $\operatorname{Ex}(\mathcal{G}, \mathcal{G}[F(v)])$ is reachable from a vertex $u \in F\left(w_{i}\right)$ for some $w_{i} \in\left\{w_{1}, \ldots, w_{m}\right\}$. Then $F\left(w_{i}\right)$ is a strongly connected component of $\mathcal{G}[F(v)] \backslash g(v)$ and $g(v)$ is not blocking in $\mathcal{G}[F(v)]$, but we chose it to be blocking.

$(\Leftarrow)$ We show that an entanglement two decomposition induces a winning strategy for two cops on $\mathcal{G}$. Observe that if a cop is on a vertex $g(v)$, for a bag $v$, and the robber is in a bag on a lower level of the tree, then the cop blocks the robber in the bags under $v$. Consider a vertex $a$ with the robber on it. Let $v$ be the bag with the smallest $F$-image (it is the lowest in the tree) among all with $a \in F(v)$ and let $v E_{T}=\left\{w_{1}, \ldots, w_{m}\right\}$, for $m \geq 0$ (if $m=0$ then $v E_{T}$ is empty). The cops wait for the robber to enter a component $\mathcal{G}\left[F\left(w_{i}\right)\right]$ or to go to $g(v)$. In the first case, they play according to the same strategy with $w_{i}$ instead of $v$. This descending along the tree is finite and on some level (w.l.o.g. already on that where $v$ is) the robber visits $g(v)$. One cop goes there. If the robber proceeds to a component $\mathcal{G}\left[F\left(w_{i}\right)\right]$, the second cop continues to chase her using the same strategy. If she leaves $F(v)$ and enters a brother bag $v^{\prime}$ of $v$, the cop from $v$ follows her there and so on until the robber is forced to go to $g(u)$, where $u$ is the predecessor of $v$. The first cop goes to $g(u)$ as well and chases the robber in this manner upwards. This process is finite and when the robber goes downwards, the second cop plays the described strategy with the difference 
that the robber cannot climb so high as before. Continuing in this way the cops finally capture the robber.

Observe that it follows that, in time $\mathrm{O}\left(n^{3}\right)$, where $n$ is the size of the input graph $\mathcal{G}$, one can not only decide whether $\mathcal{G}$ has entanglement at most two, but also compute an entanglement two decomposition of $\mathcal{G}$. The algorithm proceeds by first looking for the vertex $a_{0}$ by linear search. Then the $a_{0}$-components are computed. In every component the algorithm finds a vertex $a_{1}$ that blocks all blocking vertices of that component. If there is no such $a_{1}$, the algorithm returns "robber wins". Otherwise the procedure continues with the vertex $a_{1}$ instead of $a_{0}$ until there is no $a_{i}$-component for some $i$ (i.e. the $a_{i-1}$-component is of entanglement one). In this case the algorithm returns "Cops win" and the computed decomposition.

\section{Kelly-width for Entanglement Two}

Entanglement two decomposition of a graph leads to winning strategies for three cops in games that correspond to DAG Game and Kelly Game. The DAG Game, described in $[2,11]$, differs from the entanglement game only in these ways:

(1) The robber is infinitely fast (i.e. she can make moves along cop free paths rather than only along edges).

(2) The cops are not restricted to go to the vertex where the robber is.

(3) The robber can stay idle if no cop comes on the vertex she occupies.

$D A G$-width is the least number of cops needed to capture the robber in a monotone way, i.e. the set of vertices reachable for the robber must be monotonically decreasing.

The Kelly Game is played as the DAG Game, but the robber is invisible, i.e. the cops do not know where the robber is, and inert, i.e. the robber can move only if a cop is about to occupy the vertex where she is. Kelly-width is the least number of cops needed to capture the robber in a monotone way.

Proposition 23. For any graph $\mathcal{G}$, if $\operatorname{ent}(\mathcal{G}) \leq 2$, then the Kelly-width of $\mathcal{G}$ are at most 3 .

Proof. For illustration, we first use the entanglement two decomposition to describe a winning strategy for the cops in the DAG Game on graphs of entanglement two and then adjust this strategy to the Kelly Game. We can assume that $\mathcal{G}$ is not acyclic. Consider a entanglement two decomposition $(\mathcal{T}, F, g)$ of $\mathcal{G}$. In the DAG Game, a cop places himself on the $g$-image of the root of $\mathcal{T}$ at the beginning of a play. In general, assume that, for a bag $v$, a cop is on a blocking vertex $g(v)$ and the robber is on a vertex in $F(w)$, for a successor bag $w$ of $v$. The component $F(w)$ has also a blocking vertex $g(w)$. A cop who is not on $g(v)$ goes to $g(w)$ and the third cop visits every vertex in $F(w)$ that is not in a strongly connected component of $F(w)$. Thus the robber is forced to move down the decomposition tree and finally loses. 
The strategy of the cops in the Kelly Game is similar. Assume that a cop is on a blocking vertex $g(v)$. The cops do not know where the robber is, so they decontaminate a strongly connected component of $F(v) \backslash g(v)$ as described for the DAG Game, move a cop back on vertex $g(v)$ and continue with the next strongly connected component. Note that both winning strategies are monotone.

Proposition 23 gives the best possible upper bound for the number of cops needed to capture the robber in the same graph in the Kelly Game. Note that the third cop in the DAG Game and the Kelly Game is used to force the robber to move. Figure 6 shows a graph of entanglement two and both DAG-width and Kelly-width three, which is easy to verify.

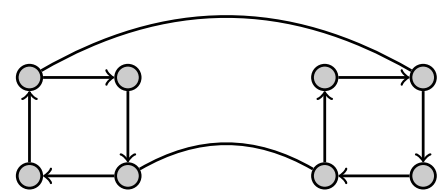

Fig. 6. A graph of entanglement 2 and both DAG-width and Kelly-width 3.

\section{Failure of a Generalisation to Entanglement $k$}

We give counterexamples to a generalisation of Proposition 15 to arbitrary number of cops. We show that, for every $k>2$, there is a graph $\mathcal{G}_{k}$ of entanglement $k$ in that, for every vertex $a$, there is a $(k-1)$-complex $a$-component. In Figure 7 such a graph is given. As the case for $k=3$ is not obvious, a counterexample graph of entanglement three is given as well (Figure 8). Circles circumscribe parts of the graph. An arrow leading to (from) a circle denotes edges to (from) all vertices in the circle. Lines without arrows denote edges in both directions. For $m>2, C_{m}$ denotes an $m$-clique.

We show first that for vertices $a_{0}, a_{1}$ and $a_{2}$ there are $(k-1)$-complex components giving corresponding strategies of the robber. Note that, for all of them, the existence of a cop free path to an exit vertex of the component is an invariant. The $a_{0}$-component $\mathcal{C}_{0}$ is induced by vertices from $T, U, B$ and the vertex $a_{2}$. The $\mathcal{C}_{0}$-strategy of the robber is to wait in $U$ until $k-1$ cops come to $U$, then proceed to $B$ and wait there for $k-1$ cops to come and so on. On the other hand, $k$ cops can expel the robber from $\mathcal{C}_{0}$.

The $a_{1}$-component $\mathcal{C}_{1}$ is induced by $a_{0}, a_{2}$ and vertices of $L, R, S$, and $F$. The $\mathcal{C}_{1}$-strategy does not use vertices of $L$. The robber waits in $S$ and $R$ (which build a $k$-clique) for $k-1$ cops to come and then goes to $F$. Three of the cops from $S \cup R$ are needed to expel her from there. Thus a path back to $S \cup R$ becomes free for the robber and she plays further as in the beginning.

The $a_{2}$-component $\mathcal{C}_{2}$ is induced by $a_{0}, T, L, R$ and $S$ whereby $R$ is not used by the robber. The $\mathcal{C}_{2}$-strategy is analogous to the $\mathcal{C}_{1}$-strategy. One can see that 


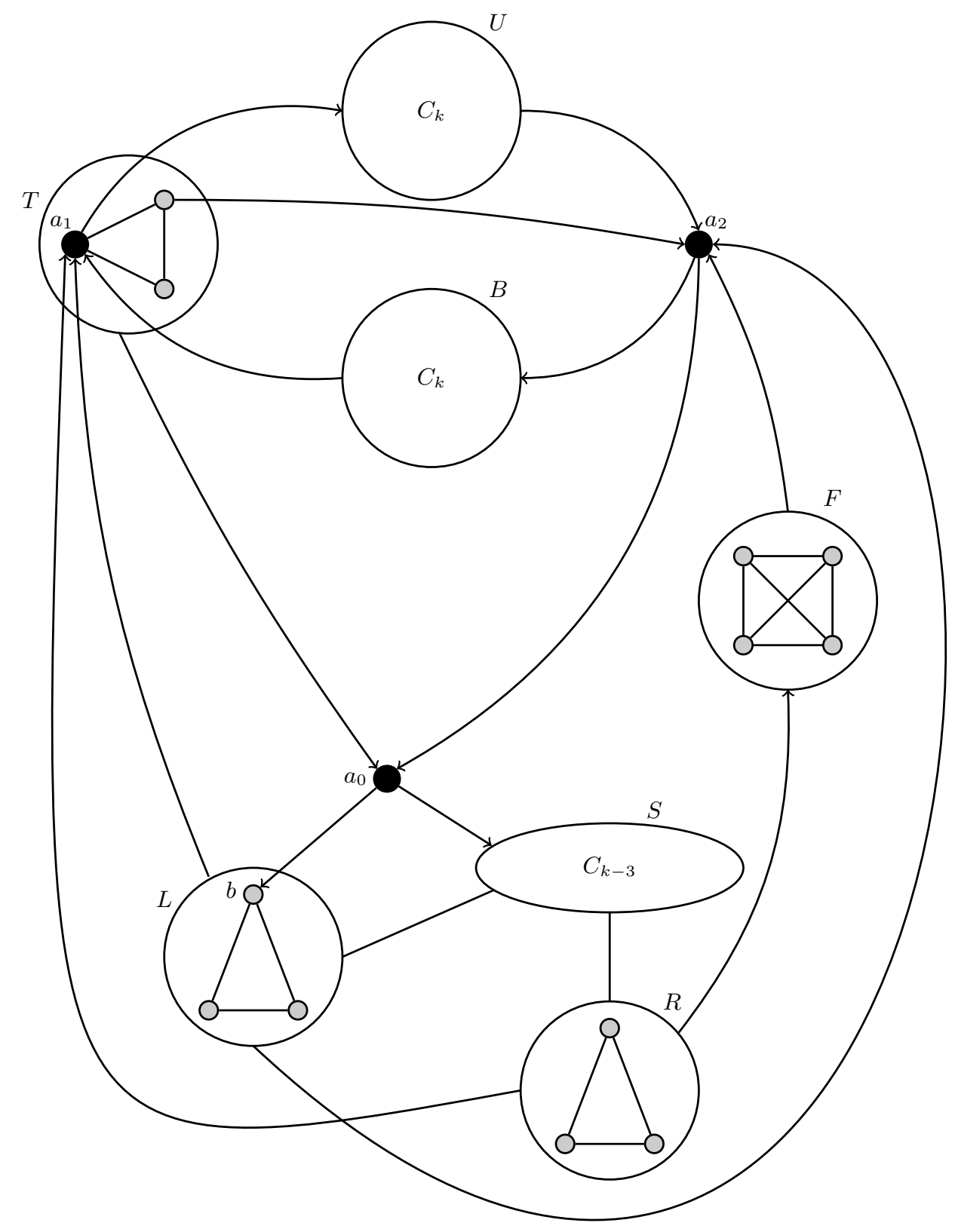

Fig. 7. A graph of entanglement $k$ with only $(k-1)$-complex components. 


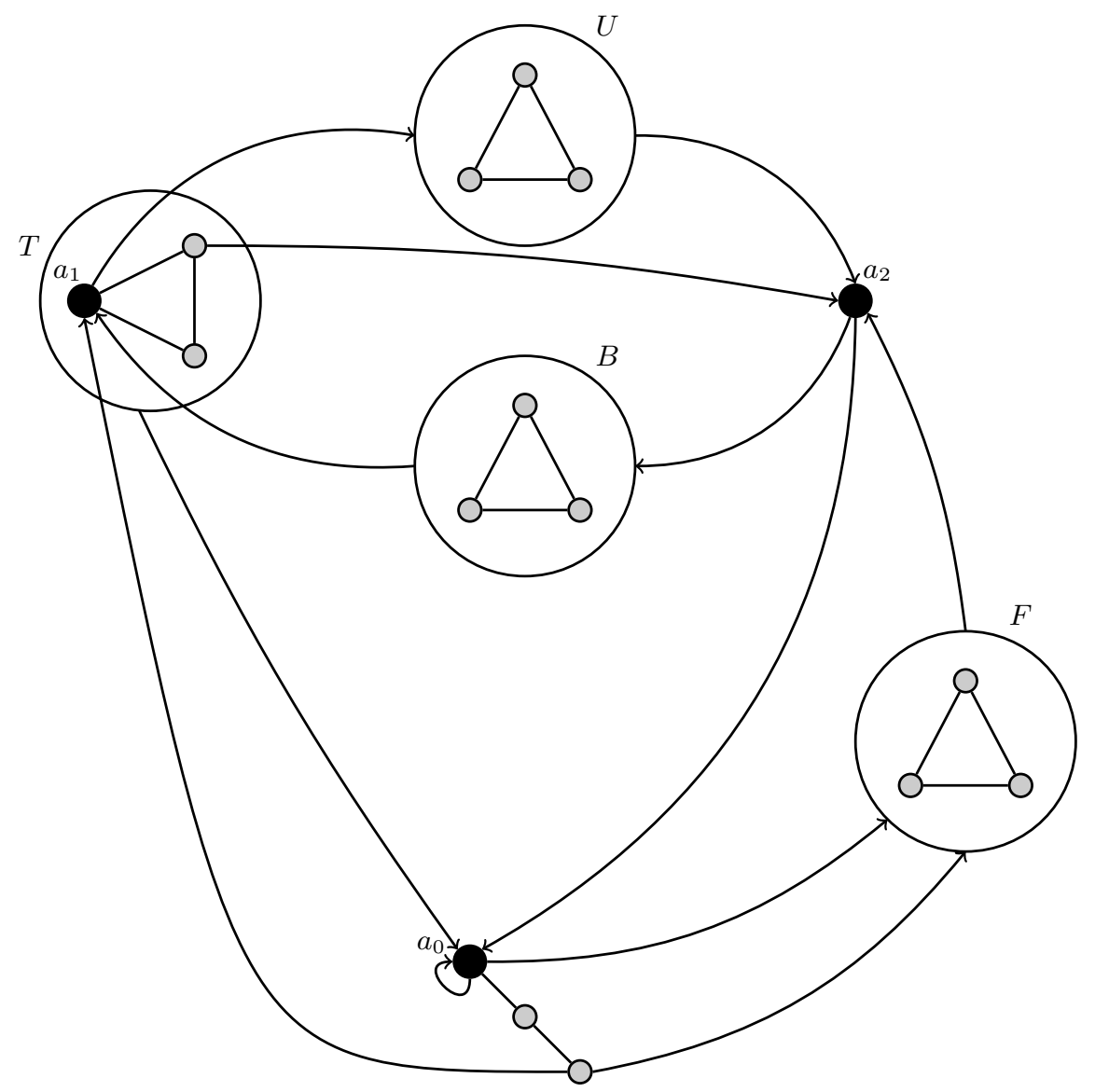

Fig. 8. A graph of entanglement 3 with only 2-complex components. 
one of the three given strategies can be used to show that, in fact, every vertex $a$ of the graph has a $(k-1)$-complex $a$-component.

Still, the entanglement of the graph is $k$. The cops have the following winning strategy in the entanglement game. One cop is placed on vertex $a_{2}$ and the robber is expelled from the component $\mathcal{C}_{0}$ defined above. If the robber visits $U$ or $F$, she is captured, because $a_{2}$ is blocked by a cop. Then $k-3$ other cops occupy vertices of $S$. If the robber goes to $R$ or to $T$, the last two cops force her to leave it, so she visits the vertex $b$. One of those two cops goes to $b$ and the other one expels the robber from $L$ and follows her to $a_{1}$. The robber must remain in $T$. In this game position, one cop is on $a_{2}$, one on $a_{1}$, one on $b$ and $k-3$ cops occupy $S$. At this time, the $k$-th cop comes from $a_{2}$ into the $a_{2}$-component $\mathcal{C}_{2}$ allowing the robber to leave it. The entanglement game in $\mathcal{C}_{2}$ with exit vertices $\operatorname{Ex}\left(\mathcal{G}, \mathcal{C}_{2}\right)$ would be lost by the cops, but they win the game on the whole graph. The cop from $a_{2}$ expels the robber from $T$. As $a_{0}$ is a dead end for her, she proceeds to $a_{2}$ and then to $B$. Then all cops except the one on $a_{1}$ capture her in $B$.

\section{References}

1. Walid Belkhir and Luigi Santocanale. Undirected graphs of entanglement 2. In Vikraman Arvind and Sanjiva Prasad, editors, Proceedings of FSTTCS'O\%, volume 4855 of $L N C S$, pages 508-519. Springer, 2007.

2. Dietmar Berwanger, Anuj Dawar, Paul Hunter, and Stephan Kreutzer. Dag-width and parity games. In Proceedings of STACS'06, volume 3884 of LNCS, pages 524536. Springer, 2006.

3. Dietmar Berwanger and Erich Grädel. Entangelement - a meausre for the complexity of directed graphs with applications to logic and games. In Proceedings of LPAR'04, volume 3452 of LNCS, pages 209-223. Springer, 2005.

4. Dietmar Berwanger, Erich Grädel, and Giacomo Lenzi. The variable hierarchy of the $\mu$-calculus is strict. Theory of Computing Systems, 40:437-466, 2007.

5. Anuj Dawar and Erich Grädel. The descriptive complexity of parity games. In Proceedings of CSL'08, volume 5213 of LNCS, pages 354-368. Springer, 2008.

6. L. C. Eggan. Transition graphs and the star-height of regular events. Michigan Math. J., 10:385-397, 1963.

7. Paul Hunter. Complexity and Infinite Games on Finite Graphs. PhD thesis, Computer Laboratory, University of Cambridge, 2007.

8. Paul Hunter and Stephan Kreutzer. Digraph measures: Kelly decompositions, games, and orderings. In Proceedings of SODA'07, pages 637-644. SIAM, 2007.

9. Thor Johnson, Neil Robertson, Paul D. Seymour, and Robin Thomas. Directed tree-width. J. Comb. Theory, Ser. B, 82(1):138-154, 2001.

10. Stephan Kreutzer and Sebastian Ordyniak. Digraph decompositions and monotonicity in digraph searching. CoRR, abs/0802.2228, 2008.

11. Jan Obdržálek. Dag-width: connectivity measure for directed graphs. In Proceedings of SODA'06, pages 814-821. ACM Press, 2006.

12. Roman Rabinovich. Complexity measures of directed graphs. Diploma thesis, RWTH-Aachen, 2008.

13. Mohammad Ali Safari. D-width, metric embedding, and their connections. $\mathrm{PhD}$ thesis, Vancouver, BC, Canada, Canada, 2007. 